$56^{\text {ème }}$ Congrès de la SFMBCB, 02014 (2011)

DOI: $10.1051 / \mathrm{sfmbcb} / 20115602014$

(C) Owned by the authors, published by EDP Sciences, 2011

\title{
Tumeur brune maxillaire révélatrice d'une hyperparathyroïdie primitive
}

\author{
Chbicheb S, El Wady W \\ Service d'Odontologie chirurgicale, CCTD, Rabat, Maroc \\ s_chbicheb@yahoo.fr
}

La tumeur brune des maxillaires est une lésion qui affecte les patients atteints d'hyperparathyroïdie. Elle apparaît comme une lésion expansive ostéolytique, touchant surtout la mandibule, les cotes, le bassin et le fémur. L'atteinte maxillaire est très rare. Elle est en rapport avec une hyperparathyroïdie primaire due à un adénome parathyroïdien dans $81 \%$ des cas, plus rarement secondaire à une insuffisance rénale chronique.

Cette tumeur est généralement douloureuse, de croissance lente, pouvant devenir agressive et destructrice. Dans la région maxillo-faciale, elle se présente comme une masse douloureuse, évidente à l'inspection, dure à la palpation, pouvant prendre un volume important et déformer ainsi l'os conduisant à une gêne masticatoire (Aoune 2000).

Une femme de 40 ans, sans antécédents médico-chirurgicaux, s'est présentée à la consultation pour une tuméfaction dans la région prémolo-molaire maxillaire droite, évoluant progressivement. L'examen clinique montre une tuméfaction de $3 \mathrm{~cm}$ dans son grand axe, indolore, recouverte par une muqueuse d'aspect normal, de consistance dure à la palpation. Les dents en regard de la tuméfaction sont saines.

Le bilan radiologique a compris une radiographie panoramique qui objective une lésion ostéocondensante, intéressant la région prémolaire et molaire droite. L'examen tomodensitométrique met en évidence une image ostéocondensante intéressant la partie antérieure et le plancher du sinus maxillaire homolatéral, avec un comblement partiel du sinus et de la cavité nasale droite.

Devant la non-spécificité de l'image radiographique, une biopsie est réalisée : elle met en un grand nombre de cellules géantes ostéoclastiques, faisant évoquer le diagnostic de tumeur brune. Le taux de PTH est de 130 ng. $1^{-1}$ (valeur normale 10- 65 ng.1 $1^{-1}$ ). L'échographie cervicale montre un adénome parathyroïdien de $2 \mathrm{~cm}$ de diamètre.

Connaissant le caractère général de cette pathologie, nous avons réalisé un bilan radiologique comprenant des radiographies du gril costal, du tibia, du péroné et de la clavicule pour exclure d'autres localisations. L'échographie abdominale est aussi normale. L'attitude thérapeutique consiste en l'ablation de l'adénome parathyroïdien, surveillance du taux de parathormone et de la tuméfaction maxillaire.

Trois mois après l'intervention, le taux de PTH est normal et la réduction de la tumeur notable, contre-indiquant ainsi tout recours à la chirurgie.

La tumeur brune serait présente dans $4.5 \%$ des cas d'hyperparathyroïdie, elle affecte surtout les cotes, le bassin, le fémur et la mandibule, avec une prédominance pour les femmes jeunes. L'atteinte maxillaire est rare et s'observe dans environ $4.5 \%$ (Aoune 2000).

L'hyperparathyroïdie primaire résulte d'un excès de production de PTH (parathormone) par un ou plusieurs adénomes, le plus souvent par une hyperplasie primaire ou rarement par une tumeur maligne des parathyrö̈des. L'hyperparathyroïdie secondaire résulte de la compensation hyperplasique, le plus souvent des quatre glandes, condition retrouvée surtout lors d'une insuffisance rénale (Goshen 2000).

$\mathrm{Au}$ niveau du squelette, l'excès d'hormone parathyroïdienne peut amener à une ostéite fibrokystique, qui est un processus de résorption diffus de l'os aboutissant à une résorption de l'os avec une rétraction de fibrose (Guney 2001). 